\title{
Band alignment at memristive metal-oxide interfaces investigated by hard x-ray photoemission spectroscopy
}

\author{
C. Lenser, ${ }^{1,}{ }^{*}$ A. Köhl, ${ }^{1}$ M. Patt, ${ }^{1}$ C. M. Schneider, ${ }^{1,2,3}$ R. Waser, ${ }^{1,2,4}$ and R. Dittmann ${ }^{1}$ \\ ${ }^{1}$ Peter Grünberg Institute, Forschungszentrum Jülich, 52425 Jülich, Germany \\ ${ }^{2}$ Research Center Jülich, JARA-FIT, 52425 Jülich, Germany \\ ${ }^{3}$ University of Duisburg-Essen, Faculty of Physics, D-47048 Duisburg, Germany \\ ${ }^{4}$ Institute of Materials in Electrical Engineering and Information Technology II, RWTH Aachen University, 52056 Aachen, Germany
}

(Received 8 July 2014; revised manuscript received 25 August 2014; published 26 September 2014)

\begin{abstract}
The electronic structure and band alignment at metal/oxide interfaces for nonvolatile memory applications are investigated by hard x-ray photoelectron spectroscopy (HAXPES) and DC transport measurements, using acceptor doped $\mathrm{SrTiO}_{3}$ as a model memristive oxide. Metal-insulator-metal (MIM) structures with a noble metal (Pt) top electrode form a Schottky barrier and exhibit rectifying properties, while a reactive metal (Ti) as top electrode shows symmetric I(V) characteristics and a flat band situation at the interface. The transition from rectifying to ohmic $\mathrm{I}(\mathrm{V})$ relations with increasing Ti thickness is discussed with respect to the electrochemical reaction at the interface, the band alignment at the electrode/oxide interface, and the slope of the energy bands across the MIM structure.
\end{abstract}

DOI: 10.1103/PhysRevB.90.115312

PACS number(s): $73.40 . \mathrm{Rw}, 73.61 . \mathrm{Ng}$

\section{INTRODUCTION}

Controlling the oxygen nonstoichiometry in transition metal oxide thin films is one of the greatest and most important challenges for nonvolatile memories based on memristive oxides [1,2]. A popular tool to engineer the redox state of the oxide is the insertion of a transition metal layer at one interface to chemically reduce the oxide and establish a controlled state of oxygen nonstoichiometry [3,4]. Such bilayer systems are very well suited to resistive switching applications [5-8].

Fe-doped $\mathrm{SrTiO}_{3}$ is used as a model material for the class of insulating perovskite oxides and can be resistively switched via an oxygen deficient filament [9-11]. While using a noble metal electrode (such as $\mathrm{Pt}$ ) results in a high initial resistance that needs to be irreversibly lowered to enable resistive switching (called electroforming) [12], the use of a reactive electrode (e.g., Ti) can remove the need for electroforming through a chemical reduction of the oxide $[8,13]$. Concomitantly to the chemical reduction, the electronic properties of an insulating oxide and especially of the metal/oxide interface can be strongly influenced by the creation of point defects as localized electronic states, or self-doping via shallow donor states close to the conduction band [14-16].

For the examination of the electronic structure and the band alignment at the interface, photoemission spectroscopy is a convenient tool since the information about the position of core level emission lines relative to the reference level (Fermi level) can be directly evaluated as a band bending in the absence of chemical changes (changes in line-shape or chemical shifts). In particular, using hard $\mathrm{x}$-ray photoemission spectroscopy (HAXPES) to evaluate band offsets is advantageous due to the large sampling depths, significantly facilitating the experiment $[3,17]$.

We have previously examined the thickness dependence of the $\mathrm{Ti}$ layer on the resistive switching properties of metal-

*c.lenser@fz-juelich.de insulator-metal (MIM) structures fabricated from epitaxial $\mathrm{SrTi}_{1-x} \mathrm{Fe}_{x} \mathrm{O}_{3-\delta}$ and demonstrated that the presence of a thin Ti layer can lead to a forming-free, bipolar switching behavior through a decreased initial resistance [13]. The mechanism of charge carrier transport through the MIM structure and band alignment at the interface remain open questions.

In this paper, we present a combinatorial analysis aimed at understanding the transition from high to low resistive $\mathrm{I}(\mathrm{V})$ characteristics in MIM structures based on $\mathrm{SrTi}_{1-x} \mathrm{Fe}_{x} \mathrm{O}_{3-\delta}$ (STFO) films with increasing thickness of the Ti top electrode. In the first step, we investigate the electronic structure of doped STO in detail, using HAXPES to determine the position of the Fermi level in the band gap (and correspondingly, the free carrier concentration in the films) for undoped as well as donor and acceptor doped material. In the second step, MIM structures with Pt and Ti electrodes are investigated by HAXPES with respect to the band alignment and the chemical states at the electrode/oxide interface, which is then correlated to the I(V) characteristics of corresponding devices to clarify the origin of the enhanced conductivity.

\section{EXPERIMENTAL}

Epitaxial $\mathrm{SrTi}_{1-x} \mathrm{Fe}_{x} \mathrm{O}_{3-\delta}$ (STFO) and $\mathrm{SrTiO}_{3}$ (STO) films were grown by pulsed laser deposition (PLD) on (001)oriented, 0.5 wt- $\%$ Nb-doped $\mathrm{SrTiO}_{3}$ single crystals (commercially available with Crystec $\mathrm{GmbH}$, Berlin, Germany). The growth conditions of all PLD films in this paper comprised a substrate temperature of $700{ }^{\circ} \mathrm{C}$, oxygen partial pressure $p \mathrm{O}_{2}=0.25 \mathrm{mbar}$, laser repetition rate of $5 \mathrm{~Hz}$ at a laser fluence of $0.8 \mathrm{~J} / \mathrm{cm}^{2}$. The film thickness was chosen to 20 $\mathrm{nm}$ for the HAXPES experiments as well as for the MIM structures, which were fabricated by sputtering a metal top electrode (Pt or Ti) onto the epitaxial films. Top electrodes were defined by optical photolithography and ion beam etching. Electrical contacting was performed using W-needles, and current-voltage characteristics were recorded with a Keithley 2611A sourcemeter, with the bottom electrode (Nb:STO) grounded and bias applied to the top electrode. 

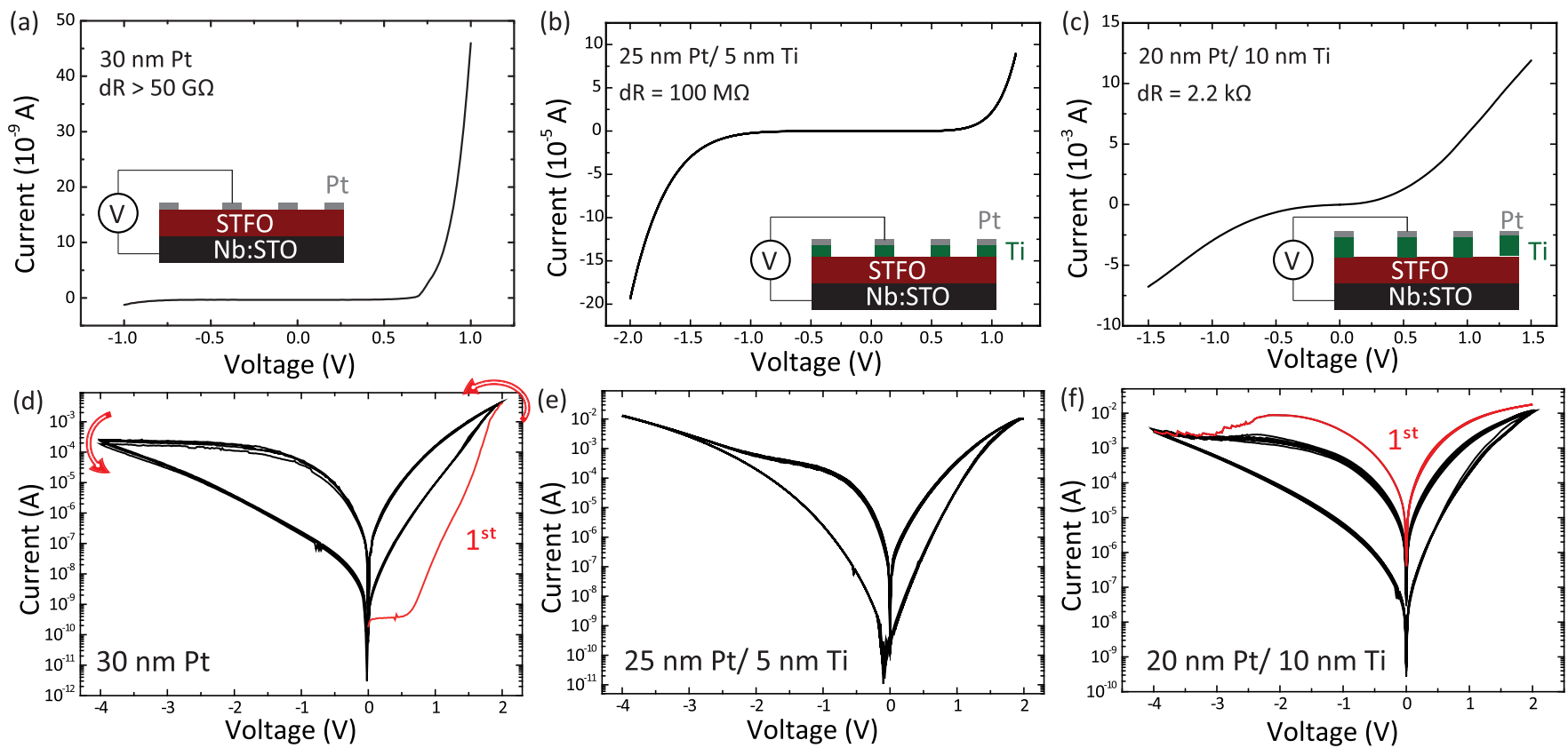

FIG. 1. (Color online) (a)-(c) I(V) characteristics of pristine MIM structures based on $20 \mathrm{~nm}$ STFO films grown on Nb:STO, with top electrodes of $30 \mathrm{~nm} \mathrm{Pt}, 25 \mathrm{~nm} \mathrm{Pt} / 5 \mathrm{~nm} \mathrm{Ti}$, and $20 \mathrm{~nm} \mathrm{Pt} / 10 \mathrm{~nm} \mathrm{Ti}$, respectively. Insets depict the sample and measurement geometry. (d)-(f): Switching curves of MIM structures corresponding to the pristine states (a), (b), and (c), respectively. The switching polarity is indicated by the red arrows in (d) and is the same for all three samples.

HAXPES experiments on the as-deposited films were performed at beamline P09 of the PETRA III synchrotron (HASYLAB, Hamburg, Germany), using a liquid $\mathrm{N}_{2}$-cooled $\mathrm{Si}$ (311) double crystal monochromator (DCM) at $5.5 \mathrm{keV}$ excitation energy. The overall energy resolution of the experiment was better than $0.35 \mathrm{eV}$, as determined from the width of the $\mathrm{Au}$ Fermi edge. The photon energy was determined before and after each sample was measured to ensure reliable calibration of the beamline. Overall, photon energy fluctuations were found to be smaller than $100 \mathrm{meV}$ over the course of 36 hours. The optical axis of the photoelectron analyzer was positioned close to the surface normal to maximize the information depth and minimize contributions from surface layers. HAXPES experiments on large arrays of MIM structures with $\mathrm{Pt}$ and $\mathrm{Pt} / \mathrm{Ti}$ electrodes close to normal emission were performed at $4.2 \mathrm{keV}$ excitation energy using a Si (111) DCM with an energy resolution better than $0.55 \mathrm{eV}$. The electrode size and separation in each array were $100 \times 100 \mu \mathrm{m}^{2}$ with $5 \mu \mathrm{m}$ separation (Pt) and $20 \times 20 \mu \mathrm{m}^{2}$ with $2 \mu \mathrm{m}$ separation $(\mathrm{Pt} / \mathrm{Ti})$.

For an excitation energy of $4.2 \mathrm{keV}$, the electron attenuation length (EAL) $\lambda$ as defined via

$$
I=I_{0} \exp (-x / \lambda)
$$

where $x$ is the overlayer thickness, and $I$ and $I_{0}$ are the signal intensity with and without overlayer, respectively, was determined to $\lambda_{\mathrm{TiO}_{x}}=53 \AA$ for a photoelectron energy of $3.9 \mathrm{keV}$ ( $\mathrm{Sr} 3 \mathrm{~d}$ core level) using $\mathrm{TiO}_{x}$ layers of varying thickness on STO. Angular dependence measurements of the Ti $2 \mathrm{p}$ photoelectron intensity on $2 \mathrm{~nm}$ thick Pt electrodes determine $\lambda_{P t}=22 \AA$ at $3.75 \mathrm{keV}$ kinetic energy of the photoelectrons. This is in good agreement with the values for the inelastic mean free path (IMFP) calculated via the TPP2M code for $\mathrm{TiO}_{2}(\lambda=61.6 \AA)$ and $\mathrm{Pt}(\lambda=32.7 \AA)$ [18]. The
IMFP value calculated for STO is $\lambda_{S T O}=65.5 \AA$, therefore we assume a similar EAL value as found for $\mathrm{TiO}_{x}$.

\section{ELECTRICAL AND MEMRISTIVE PROPERTIES}

\section{A. Initial resistance}

Figures 1(a)-1(c) show the current-voltage relations of three MIM structures with different top electrode layers in the as-deposited (pristine) state. Values of the initial (differential) resistance $d R=d V / d I$ as shown in the figures were determined by the slope of a linear fit of the current between $V= \pm 100 \mathrm{mV}$. The Pt electrode [Fig. 1(a)] shows rectifying properties with the forward direction $(\ln J \propto V)$ for positive bias at the top electrode, and the reverse direction $\left(\ln J \propto V^{1 / 2}\right)$ with negative bias at the top electrode, which is characteristic of the barrier lowering through the image-force effect encountered in Schottky junctions [19]. The initial resistance is too high to be accurately determined by a small voltage probe since the sourcemeter is not sensitive enough $\left(I<10^{-10} A\right)$, and can only be estimated to $d R>5 \times 10^{10} \Omega$.

For higher bias, we can calculate the barrier height of the rectifying $\mathrm{Pt} / \mathrm{STFO} / \mathrm{Nb}: \mathrm{STO}$ stack through the current in forward direction using the Shockley equation:

$$
J=J_{s} \exp \left(-V / n V_{T}\right), \quad J_{s}=A^{*} T^{2} \exp \left(-\phi / V_{T}\right)
$$

(with $\mathrm{V}_{T}=\mathrm{kT} / \mathrm{e}$, where $k$ is Boltzmann's constant, $\mathrm{A}^{*}$ (Richardson constant) $=156 \mathrm{~A} \mathrm{~cm}^{-2} \mathrm{~K}^{-2}, T=300 \mathrm{~K}, n$ : ideality factor, $\phi$ :barrier height). For the pristine state of the device shown in Fig. 1(a), we obtain a barrier height of $\phi=0.96 \mathrm{eV}$, and an ideality factor of $n=3.3$. For an ideal Schottky junction, $n=1$ can be expected, and the increased ideality factor of the junction hints at the presence of a 
significant contribution of tunneling currents or interface states in the system [19].

Introducing a $5 \mathrm{~nm}$ thin $\mathrm{Ti}$ layer between $\mathrm{Pt}$ and oxide lowers the initial differential resistance $d R=100 \mathrm{M} \Omega$ and removes the rectifying Schottky barrier, as evidenced by the nearly symmetric current-voltage relation shown in Fig. 1(b). The I(V) curve is almost symmetric and nonlinear, suggesting that the main contribution to the resistance arises from the volume of the STFO film through a field-accelerated transport mechanism between two slightly dissimilar interfaces. Figure 1(c) shows that the same sample geometry with a $10 \mathrm{~nm}$ Ti layer leads to a significantly smaller initial resistance $(d R=2.2 \mathrm{k} \Omega)$ and more symmetric $\mathrm{I}(\mathrm{V})$ relation.

\section{B. Resistance switching}

Devices fabricated with Pt electrodes and those with a thin $\mathrm{Ti}$ interlayer can both be resistively switched between two (or more) nonvolatile resistive states. The three exemplary switching curves shown in 1(d)-1(f) have been measured on MIM structures fabricated from the same STFO film, ensuring an equal insulator thickness for all devices. Top electrodes of $30 \mathrm{~nm} \mathrm{Pt}, 25 \mathrm{~nm} \mathrm{Pt} / 5 \mathrm{~nm} \mathrm{Ti}$, and $20 \mathrm{~nm} \mathrm{Pt} / 10 \mathrm{~nm}$ Ti [1(d)1(f), respectively] were deposited to keep the heat capacity of the top electrode comparable. The switching polarity for all marked devices is "eightwise" [9], as marked in Fig. 1(d).

The high initial resistance of the devices with $\mathrm{Pt}$ top electrodes [Fig. 1(d)] needs to be permanently lowered by a first voltage sweep (so-called electroforming), which is marked in red. Subsequently, stable switching is achieved. Using a $5 \mathrm{~nm}$ thin Ti layer [Fig. 1(e)] removes the need for this forming sweep and leaves the pristine device in the OFF state, which can be set into the ON state by a positive voltage applied to the top electrode. The switching hysteresis shows higher SET and RESET currents compared with a Pt electrode.

Increasing the thickness of the Ti layer to $10 \mathrm{~nm}$ leaves the pristine device at low initial resistance, and the device has to be reset to a high resistance state by a negative voltage (marked in red). This "reverse-forming" can typically be observed for
MIM structures fabricated from conductive metal oxides such as the $\mathrm{Ti} / \mathrm{Pr}_{1-x} \mathrm{Ca}_{x} \mathrm{MnO}_{3}$ system [20] and can be a sign of a reversible electrochemical reaction between electrode and oxide [21]. For the present case, it is a strong indication that the electrical properties of the nominally insulating STFO have been altered dramatically by the deposition of the Ti electrode.

\section{ELECTRONIC STRUCTURE OF DOPED SrTiO}

In order to determine the band alignment at the metal/oxide interface of $\mathrm{SrTi}_{0.98} \mathrm{Fe}_{x} \mathrm{O}_{3-\delta}$-based devices, the first step is the examination of the electronic structure of the films without any top electrodes using HAXPES. We investigate undoped and $2 \% \mathrm{Fe}$-doped films to glean insights into the effect of acceptor doping on the electronic properties and compare them with a donor-doped $\mathrm{Nb}$ :STO $(0.5 \mathrm{wt} \%)$ single crystal reference. The thickness of the films of $20 \mathrm{~nm}$ was chosen to avoid charging effects.

The photoemission core levels of all constituent elements display a consistent energy shift toward lower binding energies when Fe is present. Figure 2(a) shows an overview of the core levels of the major elements of the $\mathrm{SrTiO}_{3-\delta}$ and STFO. The binding energy shift has the same absolute value (within the experimental error) and sign for all core levels, for both anions and cations, and can therefore be related to a shift of the Fermi level of the Fe-doped film with respect to the undoped film. Using the Kraut method (intersection of the linear extrapolation of the leading edge of the valence band with the energy axis) to determine the valence band maximum (VBM) [22], the position of the VBM in dependence of the dopant is shown in Fig. 2(b). The degenerately donor-doped Nb:STO single crystal provides a reference of a metallically conducting material, with a charge carrier density of $n=1.57 \times 10^{20}$ $\mathrm{cm}^{-3}$ (1 electron per $\mathrm{Nb}^{5+}$ donor). We can directly calculate the position of the conduction band minimum, relative to the Fermi level, from the position of the valence band maximum and the band gap energy $\mathrm{E}_{\mathrm{Gap}}=3.25 \mathrm{eV}$ :

$$
E_{C}-E_{F}=E_{\mathrm{Gap}}-\mathrm{VBM}
$$
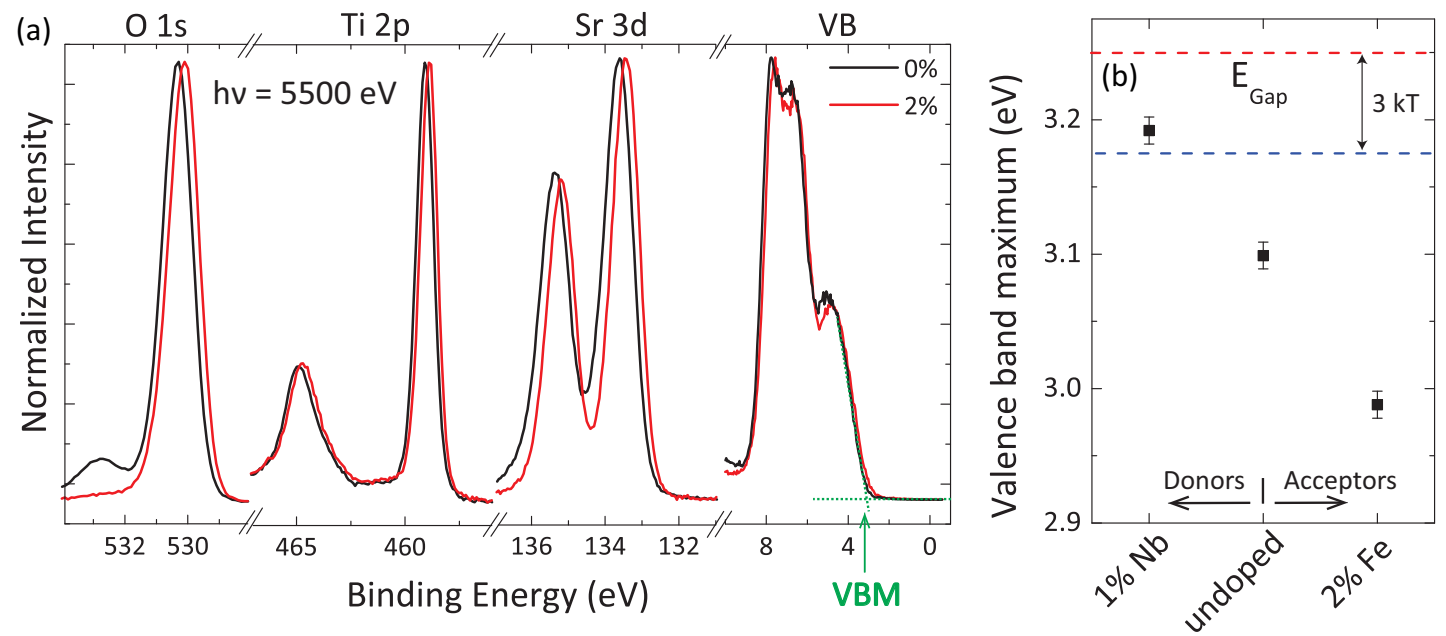

FIG. 2. (Color online) a) Core level emission lines of $\operatorname{SrTiFe}_{x} \mathrm{O}_{3-\delta}$ thin films $(x=0,0.02)$. b) Valence band maximum, as determined by the Kraut method, for different doping concentrations. The band gap energy $\mathrm{E}_{\mathrm{Gap}}=3.25 \mathrm{eV}$ and the thermal energy $3 k T=75 \mathrm{meV}$ are indicated in red and blue dashed lines, respectively. 
TABLE I. Free charge carrier concentration in doped $\mathrm{SrTiO}_{3-\delta}$.

\begin{tabular}{lcr}
\hline \hline $\mathrm{N}_{\text {Dopant }}$ & $\mathrm{E}_{C}-\mathrm{E}_{F}[\mathrm{meV}]$ & $n\left[\mathrm{~cm}^{-3}\right]$ \\
\hline $1.57 \times 10^{20} \mathrm{Nb}$ & 10 & $1.57 \times 10^{20}$ \\
undoped & 101 & $6 \times 10^{17}$ \\
$3.4 \times 10^{20} \mathrm{Fe}$ & 212 & $8 \times 10^{15}$ \\
\hline \hline
\end{tabular}

Knowing the energy separation $\mathrm{E}_{C}-\mathrm{E}_{F}$, we can calculate the carrier density in the conduction band $n$ from the integral of the Fermi distribution function $f(E, T)$ and the density of states $D(E)$ :

$$
n=\int_{E_{C}}^{\infty} D(E) \times f(E, T) d E .
$$

For sufficiently large energy separations $\mathrm{E}_{C}-\mathrm{E}_{F}>3 \mathrm{kT}$, we can substitute the Fermi distribution by the classical MaxwellBoltzmann distribution to obtain

$$
n=N_{C} \exp \left(-\frac{E_{C}-E_{F}}{k T}\right),
$$

where $N_{C}$ is the effective density of states in the conduction band, which has been determined by Moos et al. to be [23]

$$
N_{C}(T)=4.1 \times 10^{16} \mathrm{~cm}^{-3}(T / K)^{3 / 2} .
$$

At $300 \mathrm{~K}$, we obtain values of $N_{C}=2.13 \times 10^{20} \mathrm{~cm}^{-3}$ and $3 \mathrm{kT}=75 \mathrm{meV}$. Inserting the values of VBM shown in Fig. 2(b) into Eq. (5), we obtain a strong variation of the charge carrier density $n$ (occupied density of states) as a function of the dopant, as tabulated in Table I. Note that since $\mathrm{E}_{C}-\mathrm{E}_{F}<3 \mathrm{kT}$ for $\mathrm{Nb}$-doped $\mathrm{SrTiO}_{3}$, Eq. (5) is invalid, and we assume one electron per $\mathrm{Nb}$ atom to estimate $n$, following the general assumption of electronic compensation of the $\mathrm{Nb}$ dopants $(n \approx[N b])$.

Concerning the error propagation in this analysis, we note that the exponential relation $n \sim \exp \left(E_{C}-E_{F}\right)$ leads to a large uncertainty in $n$ for relatively small uncertainties in $E_{C}-$ $E_{F}$. A change of $50 \mathrm{meV}$ in $E_{C}-E_{F}$ can lead to almost an order of magnitude change in $n$. The absolute values of $n$ in Table I should therefore be viewed with caution. However, the difference between STO and STFO is consistently found in all core levels and is too large to be a result of external error sources.

We would like to add a brief explanatory note on the charge compensation of the point defect in the films, since the concentrations of free carriers are high for a material that is insulating in the bulk. While the STFO films are nominally acceptor doped, an abundance of oxygen vacancies $\mathrm{V}_{o}^{\bullet \bullet}$ is introduced into the film during PLD growth. The kinetic constraints that dominate PLD growth can induce point defect configurations in the films that are not encountered in bulk materials produced in thermal equilibrium [24-27]. In addition, space charge effects induced by the proximity to the surface can play a role in determining the concentration of free carriers in the film [28].

As a result, we find that undoped $\mathrm{SrTiO}_{3}$ films have unexpectedly high concentrations of free carriers, as evidenced by the close proximity of the Fermi level to the bottom of the conduction band. However, intentional acceptor doping reduces the amount of free charge carriers by trapping the free carriers induced through $\mathrm{V}_{O}^{\bullet}$ into in-gap states on the $\mathrm{Fe}$ ions. We find evidence for this behavior also in the presence of a mixed $\mathrm{Fe}^{2+} / \mathrm{Fe}^{3+}$ (and absence of $\mathrm{Fe}^{4+}$ ) valence state in the near-surface region of such films $[29,30]$. We therefore deal with $n$-doped films even when admixing substantial amounts of acceptor-type cations.

\section{INTERFACE REACTION}

Before examining the band alignment at the Ti/STFO interface, it is worthwhile to consider the chemical state of the interface. We show above that increasing the Ti thickness leads to decreased resistance values if the STFO thickness is kept constant. Figures 3(a) and 3(b) demonstrate that keeping the $\mathrm{Ti}$ thickness constant and varying the thickness of the STFO layer produces the same effect: Thinner STFO films have dramatically reduced resistance in the pristine state. In fact, a $5 \mathrm{~nm}$ Ti layer deposited on a $10 \mathrm{~nm}$ STFO film produces electrically shorted MIM structures that cannot be resistively switched.

The fact that the initial resistance can be controlled by the variation of both top electrode and insulator thickness is important when examining such layer systems with HAXPES. A variation of the top electrode thickness does not only change the resistance state of the sample but also the information depth of the photoemission experiment. Since this is not trivial to account for, keeping the thickness of the top electrodes constant and varying the insulator thickness facilitates the direct comparison of photoemission spectra. The Pt capping layer thickness of $2 \mathrm{~nm}$ corresponds roughly to $\lambda_{P t}=22 \AA$, therefore the photoemission spectra probe approximately 10 $\mathrm{nm}$ into the $\mathrm{TiO}_{x} / \mathrm{STO}$ underneath the Pt, assuming an EAL value of $50 \AA$ (see Sec. II).

The Ti $2 p$ photoemission spectra shown in Fig. 3(c) shed light on this thickness dependence and the chemical state at the interface. The Ti $2 p$ spectrum of a $2 \mathrm{~nm}$ Pt electrode on a $20 \mathrm{~nm}$ STFO film (black) serves as a reference for a single, spin-orbit split doublet corresponding to fully oxidized $\mathrm{Ti}^{4+}$, while the Ti 2 p spectrum of a Nb:STO $(0.5 \mathrm{wt} \%)$ single crystal (green) demonstrates that the observed changes are not related to the substrate.

Inserting a $4 \mathrm{~nm}$ thin $\mathrm{Ti}$ layer (red line) results in a low binding energy shoulder, indicating the presence of $\mathrm{Ti}$ suboxides in the probed volume. Since the information depth is greater than the $\mathrm{TiO}_{x}$ layer thickness at an electron kinetic energy of $3.7 \mathrm{keV}$ (corresponding to an electron attenuation length $\lambda \approx 53 \AA$ ), the contribution of Ti electrode and STFO are indistinguishable. However, the presence of suboxides, along with the decreased electrical resistance, points to a reduction of the STFO film by the Ti electrode, which is oxidized in turn. A detailed analysis of the spectrum (provided in Ref. [13]) reveals that the spectral envelope can only be reproduced by including one component for each possible oxidation state of $\mathrm{Ti}^{x+}$, excepting the metallic state $(x=$ $1,2,3,4)$.

Decreasing the insulator thickness to $10 \mathrm{~nm}$ (blue line) results in a much more pronounced structure on the low binding energy side of the Ti $2 p$ doublet. Since the probed volume is the same for both Pt/Ti/STFO samples, we conclude that a 

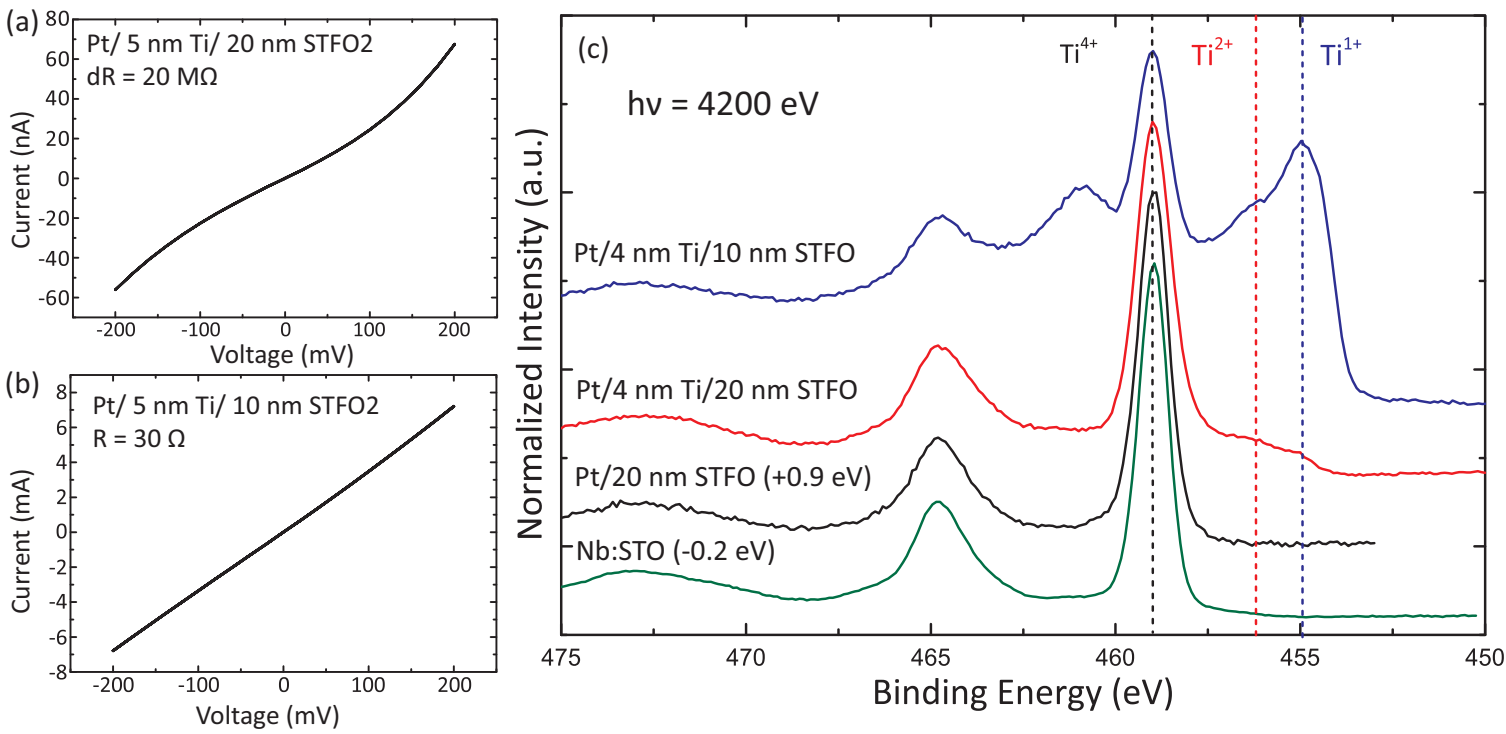

FIG. 3. (Color online) (a), (b): I(V) characteristics of two MIM devices in the pristine state, based on a $20 \mathrm{~nm}$ and a $10 \mathrm{~nm}$ STFO film,

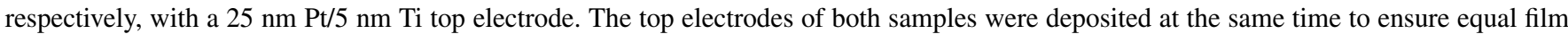
thickness. (c) Ti $2 \mathrm{p}$ core level spectra recorded on samples with $2 \mathrm{~nm} \mathrm{Pt} / 4 \mathrm{~nm}$ Ti electrodes, deposited on a $10 \mathrm{~nm} \mathrm{SrTi}_{0.95} \mathrm{Fe}_{0.05} \mathrm{O}_{3-\delta}\left(\mathrm{STFO}_{5}\right)$ and $20 \mathrm{~nm}$ STFO film (blue and red, respectively), plus a $2 \mathrm{~nm} \mathrm{Pt} / 20 \mathrm{~nm}$ STFO sample (black line) and a Nb:STO (0.5 wt\%) single crystal for reference. The Pt/STFO spectrum was shifted in energy by $+0.9 \mathrm{eV}$, and the $\mathrm{Nb}: \mathrm{STO}$ by $-0.2 \mathrm{eV}$ to facilitate the comparison of the spectra.

thinner STFO layer underneath the Ti layer results in a larger amount of Ti suboxides. Note that while the blue spectrum is recorded on a stack based on STFO with a slightly different $\mathrm{Fe}$ concentration (5\%), we do not expect a qualitative difference to $2 \% \mathrm{Fe}$.

From the increased amount of Ti suboxides with decreased STFO thickness, in conjunction with the lowered electrical resistance, we conclude that oxygen ions move from the bulk of the STFO film into the Ti electrode, which induces electrical conductivity in the STFO through the self-doping with oxygen vacancies $\mathrm{V}_{O}^{\bullet \bullet}$. The driving force for this movement is a result of the difference in the electrochemical potential of oxygen in metallic Ti and STFO. The amount of suboxides present is therefore indicative of the resistive state. In addition, the thickness dependence leads us to consider that the STFO film serves as a finite reservoir for oxygen that can be transferred in a redox reaction to the Ti layer. A thinner Ti layer seems to be less effective at reducing the STFO than a thicker one, as evidenced by the resistance dependence on Ti thickness, and correspondingly a thinner STFO film is more heavily reduced than a thicker one. For this reason, the amount of reduction in the STFO film - and thereby also the electrical conductivity - can be controlled by the thickness of the $\mathrm{Ti}$ layer.

\section{BAND ALIGNMENT}

\section{A. Rectifying interfaces}

To examine the band offset at the Pt/STFO interface, we performed HAXPES on fabricated MIM structures using $2 \mathrm{~nm}$ thin Pt electrodes to minimize the absorption of photoelectrons in the Pt overlayer. The procedure to evaluate band offsets is outlined in Fig 4: The energy positions of the respective core level emission lines of the two interfacing materials (in this case, Pt and STFO) were established on reference samples, for which we used a $40 \mathrm{~nm}$ Pt layer sputtered onto a (001)-oriented $\mathrm{Nb}: \mathrm{SrTiO}_{3}$ crystal as a reference for the energy position of the $\mathrm{Pt} 4 \mathrm{p}_{3 / 2}$ line (blue lines), and the STFO thin

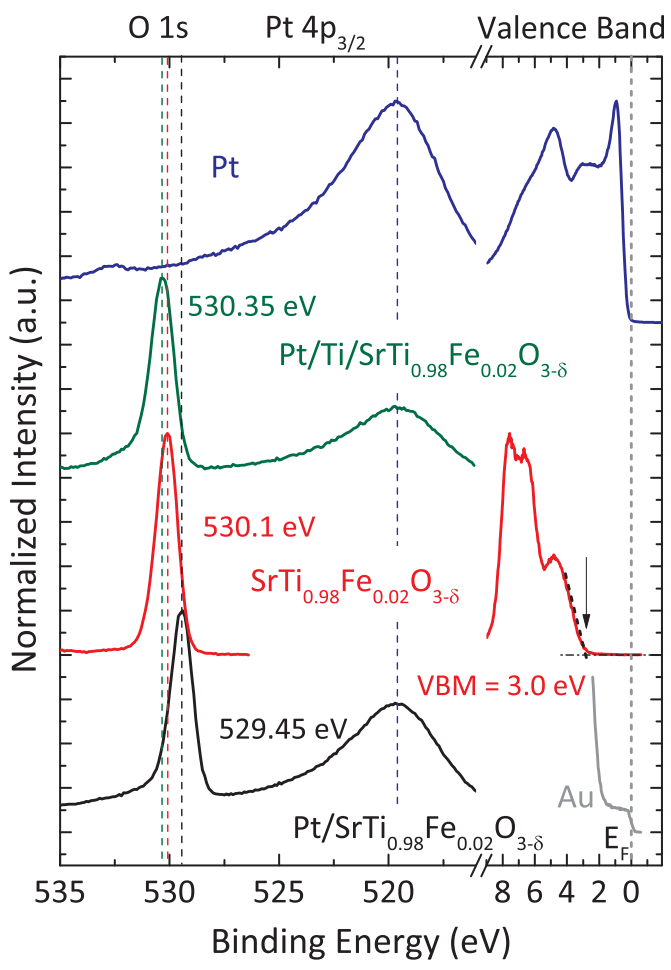

FIG. 4. (Color online) Core level and valence band emission from the Pt reference (blue), the STFO reference (red), the $2 \mathrm{~nm}$ $\mathrm{Pt} / \mathrm{STFO}$ interface (black), and the $2 \mathrm{~nm} \mathrm{Pt} / 4 \mathrm{~nm}$ Ti /STFO (green). The Au Fermi edge for photon energy calibration is shown in gray. 
film discussed above as a reference for the $\mathrm{O} 1 \mathrm{~s}$ (red lines), Ti $2 p$ and $\mathrm{Sr} 3 \mathrm{~d}$ core level emission lines. The position of the valence band maximum (VBM) of STFO is determined by linear extrapolation of the leading edge to the energy axis [22].

These reference spectra are then compared to photoemission spectra of a $2 \mathrm{~nm}$ thin layer of $\mathrm{Pt}$, sputter-deposited under the same conditions as the Pt reference, on a $20 \mathrm{~nm}$ epitaxial STFO film, grown on a (001)-oriented $\mathrm{Nb}: \mathrm{SrTiO}_{3}$ crystal under identical conditions as the STFO reference (black line). The relative distance between these emission lines can then be evaluated to determine the valence band offset (VBO) at the interface:

$$
\begin{aligned}
\mathrm{VBO}= & \left(E_{O 1 s}-E_{P t 4 p_{3 / 2}}\right)_{\text {interface }}+\left(E_{P t 4 p_{3 / 2}}-E_{F}\right)_{P t} \\
& -\left(E_{O 1 s}-E_{\mathrm{VBM}}\right)_{\mathrm{STO}}
\end{aligned}
$$

or since the Pt core level is referenced to the Fermi level, which is the reference level for photoemission $\left(\mathrm{E}_{F}=0\right)$,

$$
\mathrm{VBO}=E_{O 1 s, \text { interface }}-\left(E_{O 1 s}-E_{\mathrm{VBM}}\right)_{\mathrm{STO}} .
$$

To certify that the observed effect is due to a band offset (i.e., a shift of the oxide electronic structure relative to the Fermi level of the system), the observed shift has to be visible in all core levels, which is indeed what we find.

While Eq. (7) can be applied for any given set of core levels of the oxide and the metal, using the $\mathrm{O}$ 1s and $\mathrm{Pt} 4 \mathrm{p}_{3 / 2}$ lines improves the reliability of the method and comparability between samples since the difference $E_{O 1 s}-E_{P t 4 p_{3 / 2}}$ can be measured in the same spectrum. Applying Eq. (8) to the energy positions extracted from the spectra displayed in Fig. 3, we calculate a valence band offset of $2.35 \mathrm{eV}$ for the $2 \mathrm{~nm}$ Pt/STFO structure, which corresponds to a conduction band offset $(\mathrm{CBO})$ of $\phi_{X P S}=0.9 \mathrm{eV}$ when assuming a band gap of $3.25 \mathrm{eV}$, in good agreement with the calculated barrier height of $\phi=0.96 \mathrm{eV}$ from charge carrier transport. However, a slight underestimation of the barrier height determined via photoemission could be an artifact of an x-ray induced surface photovoltage. Investigations of the barrier height of $\mathrm{Pt} / \mathrm{Ba}_{1-x} \mathrm{Sr}_{x} \mathrm{TiO}_{3}$ interfaces yielded comparable values [31].

\section{B. Symmetric interfaces}

The rectifying Pt/STFO interface serves as a point of reference for the investigation of the symmetric interfaces obtained when Ti is used as the top electrode material. We used the same HAXPES technique described above to investigate the band alignment at the Ti/STFO interface. Figure 4 contains the $\mathrm{Pt}$ $4 \mathrm{p}_{3 / 2}$ and $\mathrm{O} 1 \mathrm{~s}$ spectra of the sample fabricated from a $20 \mathrm{~nm}$ epitaxial STFO PLD film with a $4 \mathrm{~nm}$ Ti top electrode, capped by a $2 \mathrm{~nm}$ layer of $\mathrm{Pt}$ (green line). In contrast to the rectifying $\mathrm{Pt} / \mathrm{STFO}$ interface (black line in Fig. 4), the presence of a Ti interlayer causes a shift of the $\mathrm{O} 1$ s core level to higher binding energies, without any significant changes in line shape. The same shifts can be found in the Sr $3 \mathrm{~d}$ and Ti $2 \mathrm{p}$ lines (data not shown).

When treating the energy position of the $\mathrm{O} 1$ s emission line of the $4 \mathrm{~nm} \mathrm{Ti} / 20 \mathrm{~nm}$ STFO structures with the formalism given by Eq. (7), we obtain a $\mathrm{VBO}=3.25 \mathrm{eV}$. This value is identical to the assumed band-gap energy $3.25 \mathrm{eV}$, implying a flat band situation with $\mathrm{CBO}=0 \mathrm{eV}$. However, given the small uncertainty imposed by the experimental resolution and possible small photon energy fluctuations in the range of $30-50 \mathrm{meV}$, we include the $\mathrm{I}(\mathrm{V})$ characteristics shown in Fig. 1(b) into our considerations. Since Ti/STFO MIM structures with a Ti thickness of $\mathrm{d}_{T i} \leqslant 5 \mathrm{~nm}$ are generally neither shorted nor rectifying, we conclude that the VBO obtained from HAXPES denotes a position of the Fermi level close below the conduction band.

\section{DISCUSSION}

The conclusions of the transport studies and the photoemission experiments are summarized in Fig. 5, schematically showing band diagrams for the (a) Pt/STFO/Nb:STO and (b) $\mathrm{Pt} / \mathrm{TiO}_{x} / \mathrm{STFO} / \mathrm{Nb}: \mathrm{STO}$ systems. The Pt/STFO interface is characterized by an energy barrier, yielding rectifying $\mathrm{I}(\mathrm{V})$ relations that can be described by thermionic field emission over a Schottky barrier. The barrier height can be estimated to be $\phi=0.96 \mathrm{eV}$ from Eq. (2) or $0.9 \mathrm{eV}$ from photoemission.

An important consideration can be made regarding the width of the depletion layer in the STFO film when the Debye length is used to estimate the decay of the electric field caused by the built-in potential:

$$
L_{D}=\sqrt{\epsilon_{0} \epsilon_{r} k T / 2 e^{2} n_{0}},
$$
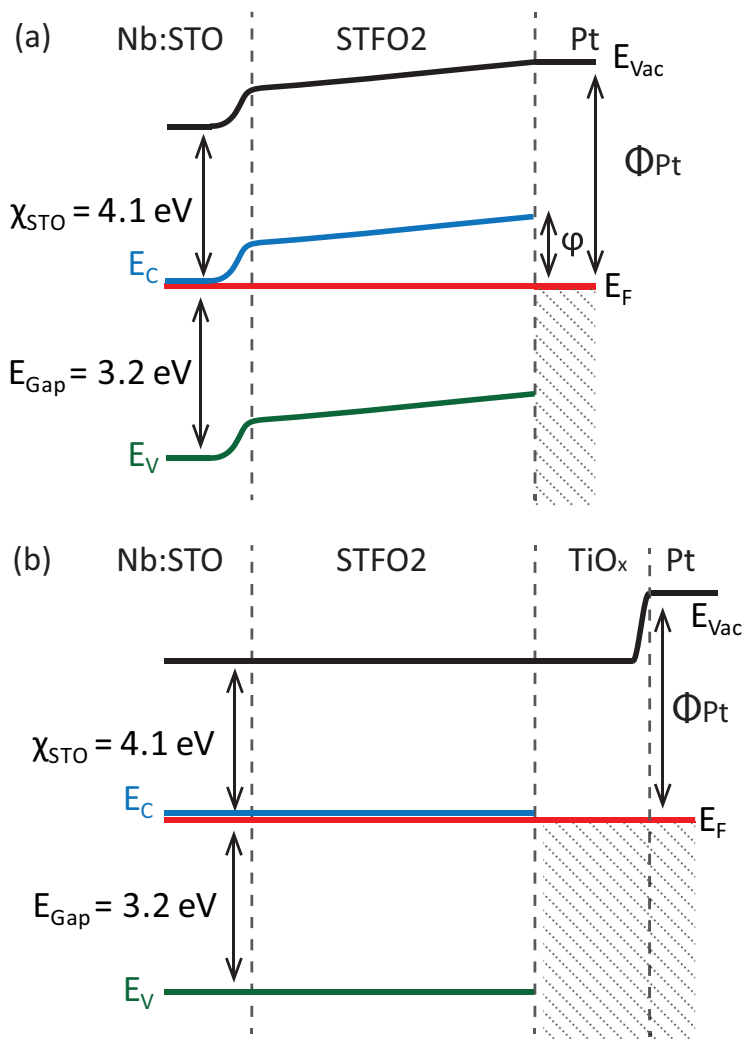

FIG. 5. (Color online) Schematic band diagram for (a) Pt/STFO and (b) $\mathrm{Pt} / \mathrm{TiO}_{x} / \mathrm{STFO}$ interface with a thin Ti interlayer, respectively. The work function of $\mathrm{Pt}$ is $\Phi_{P t}=5.65 \mathrm{eV}$, and the electron affinity of STO is $\chi_{\text {STO }}=4.1 \mathrm{eV}$. 
where $\epsilon_{0}$ is the permittivity of vacuum, $\epsilon_{r}$ is the dielectric constant, and $k$ is Boltzmann's constant. Using the value of $n_{0} \approx 8 \times 10^{15} \mathrm{~cm}^{-3}$ determined for $\mathrm{SrTi}_{0.98} \mathrm{Fe}_{0.02} \mathrm{O}_{3-\delta}$ by HAXPES and the dielectric constant of bulk $\mathrm{SrTiO}_{3}, \epsilon_{r}=300$, we obtain $L_{D} \approx 164 \mathrm{~nm}$. Allowing for a decreased dielectric constant $\epsilon_{r}$ due to chemical doping or the thin film geometry $[32,33]$, we note that the Debye length remains much larger than the film thickness for reasonable variation of $\epsilon_{r}$ due to the square-root relation between $L_{D}$ and $\epsilon_{r}$.

Since the extension of the space-charge region $w_{s c}$ is itself larger than $L_{D}\left(w_{s c} \sim 8-10 \times L_{D}\right)$ [19], we see that $w_{s c}$ $\gg 20 \mathrm{~nm}$, so that the STFO film is too thin to shield the built-in potential of the barrier. The main potential drop is then located at the interface to the $\mathrm{Nb}$ :STO bottom electrode, where $L_{D}=1.1 \mathrm{~nm}\left(n_{0}=1.7 \times 10^{20} \mathrm{~cm}^{-3}\right)$ is much smaller, causing the electric field induced by the built-in potential to decay quickly in the Nb:STO bottom electrode. This is reflected by the schematic slope of the energy bands in Fig. 5(a).

The schematic band diagram of $\mathrm{Pt} / \mathrm{TiO}_{x} / \mathrm{STFO} / \mathrm{Nb}: \mathrm{STO}$ with a thin $\mathrm{Ti}$ interlayer is shown in Fig. 5(b). The band alignment obtained from the HAXPES experiments indicates a flat band situation, in agreement with the removal of the Schottky barrier. The Ti top electrode scavenges oxygen from the epitaxial oxide and forms a mixture of suboxides, as can be seen in the photoemission spectra in Sec. V. Since most Ti suboxides show electrical conductivity, the electrode does not necessarily govern the electrical properties of the stack. However, the actual oxidation state of the Ti at the interface with $\mathrm{Pt}$ is unknown at this point. We therefore assume $\mathrm{TiO}_{x}$ to be metallic in nature for this band scheme, which is reflected by the steep slope of $\mathrm{E}_{V a c}$ at the $\mathrm{Pt} / \mathrm{TiO}_{x}$ interface in Fig. 5(b). The essential point is the absence of an energy barrier.

The resistivity of this stack is either determined by (i) the actual position of the Fermi level relative to the conduction band minimum in the STFO or by (ii) the oxidation state of the $\mathrm{TiO}_{x}$ electrode. In case (i), the position of the Fermi level is governed by the amount of self-doping by oxygen vacancies, which are introduced into the material through chemical reduction by the Ti electrode. Depending on the doping level, the conduction band is either within $3 k T$ of $\mathrm{E}_{F}$, resulting in the linear $\mathrm{I}(\mathrm{V})$ behavior of a degenerately doped semiconductor, or $\mathrm{E}_{C}-\mathrm{E}_{F}>3 k T$, in which case the STFO acts as a semiconductor or insulator. We can estimate the concentration of oxygen vacancies needed to form impurity bands, which can induce degeneracy, using the effective Bohr radius $a^{*}$ :

$$
a^{*}=\frac{m_{0} \epsilon_{r} a_{0}}{m^{*}} .
$$

Using $\epsilon_{r}=300$ and an effective mass of $m^{*} / m_{0}=1.8$ [34], we obtain an effective Bohr radius of $a^{*}=8.8 \mathrm{~nm}$. Since the effective Bohr radius is a measure for the extension of an impurity wave function, we can estimate the concentration of donor impurities necessary to form impurity bands by

$$
N_{D}^{*} \approx\left(1 / a^{*}\right)^{3}=1.5 \times 10^{18} \mathrm{~cm}^{-3} .
$$

We note that a decreased $\epsilon_{r}$ value can strongly affect $N_{D}^{*}$ due to the cubic relation between $\epsilon_{r}$ and $N_{D}^{*}$. More detailed investigations are necessary to evaluate this problem, however, but this may be a reason why oxygen vacancy-related impurity bands are seldom observed in STO thin films.

As we show in Sec. V, the amount of oxygen transferred from STFO to Ti depends on the thickness of both materials. By controlling the $\mathrm{Ti}$ thickness, the electronic state of a given STFO film can thus be varied from insulating behavior to a degenerately doped semiconductor.

It is also conceivable that the $\mathrm{Ti}$ oxidation state of the electrode determines the stack resistance [case (ii)]. This is an extreme case since the resistance of the STFO needs to be much lower than that of the electrode. However, this possibility should not be ruled out, especially for thick Ti layers and thin STFO films, resulting in a strong reduction of the insulator and strong oxidation of the metal. In fact, this scenario is the most likely reason for the observation of "reverseforming" (i.e., increase of a low initial resistance) as shown in Fig. 1(f).

The transition from rectifying to conducting properties is accompanied by a change in the mechanism of charge carrier transport, as evidenced by the shape of the I(V) curves. However, we note that a variety of charge transport mechanisms have been discussed for electrically treated $\mathrm{SrTiO}_{3}$, including resonant tunneling [35], polaronic conduction [36,37], and space-charge limited conduction (SCLC) [38]. All of the above (and others) exhibit nonlinear charge transport as a function voltage. It therefore goes far beyond the scope of this paper to discern which mechanism is prevalent in $\mathrm{Pt} / \mathrm{TiO}_{x} / \mathrm{STFO} / \mathrm{Nb}: \mathrm{STO}$ stacks, and we restrict ourselves to the observation of a transition from insulating to conducting properties with increasing $\mathrm{Ti}$ thickness.

In conclusion, we have demonstrated the analysis of the band alignment at metal/oxide interfaces with rectifying, insulating, and ohmic character. The respective conduction behavior can be interpreted in terms of an alignment of electronic levels at the metal/oxide interface, which can be quantified based on HAXPES experiments, complemented by DC transport measurements. These observations are particularly important with regard to memristive systems built from metal/oxide interfaces, since it is important to understand that the relative thickness of metal and oxide determines not only the oxygen nonstoichiometry of the oxide, but can concomitantly influence the charge carrier transport as well.

\section{ACKNOWLEDGMENTS}

The authors would like to acknowledge the beamtime awarded under proposal number I-20100325, and thank A. Gloskovskii and S. Thiess for assistance with the HAXPES experiments. The authors gratefully acknowledge financial support by the Deutsche Forschungsgemeinschaft (SFB 917), and C.L. gratefully acknowledges financial support by the EC FP7 MATERA project "Functional materials for resistive switching memories" (FMRSM) and the NRW-EU Ziel 2Program. 
[1] R. Waser, R. Dittmann, G. Staikov, and K. Szot, Adv. Mater. 21, 2632 (2009).

[2] A. Sawa, Materials Today 11, 28 (2008).

[3] M. Sowinska, T. Bertaud, D. Walczyk, S. Thiess, M. A. Schubert, M. Lukosius, W. Drube, C. Walczyk, and T. Schroeder, Appl. Phys. Lett. 100, 233509 (2012).

[4] R. Yasuhara, T. Yamamoto, I. Ohkuba, H. Kumigashira, and M. Oshima, Appl. Phys. Lett. 97, 132111 (2010).

[5] A. Sawa, T. Fujii, M. Kawasaki, and Y. Tokura, Appl. Phys. Lett. 85, 4073 (2004).

[6] M. Lee, C. B. Lee, D. Lee, S. R. Lee, M. Chang, J. H. Hur, Y. Kim, C. Kim, D. H. Seo, S. Seo, U. Chung, I. Yoo, and K. Kim, Nat. Mater. 10, 625 (2011).

[7] B. Govoreanu, G. S. Kar, Y.-Y. Chen, V. Paraschiv, S. Kubicek, A. Fantini, I. P. Radu, L. Goux, S. Clima, R. Degraeve, N. Jossart, O. Richard, T. Vandeweyer, K. Seo, P. Hendrickx, G. Pourtois, H. Bender, L. Altimime, D. J. Wouters, J. A. Kittl, and M. Jurczak, in 2011 IEEE International Electron Devices Meeting - IEDM '11 (IEDM Tech. Dig., 2011), p. S31.6.

[8] J. P. Strachan, J. J. Yang, L. A. Montoro, C. A. Ospina, A. J. Ramirez, A. L. D. Kilcoyne, G. Medeiros-Ribeiro, and R. S. Williams, Beilstein J. Nanotechnol. 4, 467 (2013).

[9] R. Muenstermann, T. Menke, R. Dittmann, and R. Waser, Adv. Mater. 22, 4819 (2010).

[10] C. Lenser, A. Kuzmin, J. Purans, A. Kalinko, R. Waser, and R. Dittmann, J. Appl. Phys. 111, 076101 (2012).

[11] A. Koehl, H. Wasmund, A. Herpers, P. Guttmann, S.Werner, K. Henzler, H. Du, J. Mayer, R. Waser, and R. Dittmann, APL Mater. 1, 042102 (2013).

[12] T. Menke, R. Dittmann, P. Meuffels, K. Szot, and R. Waser, J. Appl. Phys. 106, 114507 (2009).

[13] S. Stille, C. Lenser, R. Dittmann, A. Koehl, I. Krug, R. Muenstermann, J. Perlich, C. Schneider, U. Klemradt, and R. Waser, Appl. Phys. Lett. 100, 223503 (2012).

[14] J. J. Yang, D. B. Strukov, and D. R. Stewart, Nat. Nanotechnol. 8, 13 (2013).

[15] A. Padovani, L. Larcher, P. Padovani, C. Cagli, and B. D. Salvo, 2012 4th IEEE International Memory Workshop (IEEE, Milan, 2012).

[16] S. U. Sharath, T. Bertaud, J. Kurian, E. Hildebrandt, C. Walczyk, P. Calka, P. Zaumseil, M. Sowinska, D. Walczyk, A. Gloskovskii, T. Schroeder, and L. Alff, Appl. Phys. Lett. 104, 063502 (2014).

[17] A. Zenkevich, R. Mantovan, M. Fanciulli, M. Minnekaev, Y. Matveyev, Y. Lebedinskii, S. Thiess, and W. Drube, Appl. Phys. Lett. 99, 182905 (2011).
[18] S. Tanuma, C. Powell, and D. Penn, Surf. Interface Anal. 21, 165 (1994).

[19] S. Sze, Physics of Semiconductor Devices (Wiley, New York, 1981).

[20] F. Borgatti, C. Park, A. Herpers, F. Offi, R. Egoavil, Y. Yamashita, A. Yang, M. Kobata, K. Kobayashi, J. Verbeeck, G. Panaccione, and R. Dittmann, Nanoscale 5, 3954 (2013).

[21] A. Herpers, C. Lenser, C. Park, F. Offi, F. Borgatti, G. Panaccione, S. Menzel, R. Waser, and R. Dittmann, Adv. Mater. 26, 2730 (2014).

[22] E. A. Kraut, R. W. Grant, J. R. Waldrop, and S. P. Kowalczyk, Phys. Rev. Lett. 44, 1620 (1980).

[23] R. Moosand K. Haerdtl, J. Am. Ceram. Soc. 80, 2549 (1997).

[24] T. Ohnishi, M. Lippmaa, T. Yamamoto, S. Meguro, and H. Koinuma, Appl. Phys. Lett. 87, 241919 (2005).

[25] T. Ohnishi, K. Shibuya, T. Yamamoto, and M. Lippmaa, J. Appl. Phys. 103, 103703 (2008).

[26] D. J. Keeble, S. Wicklein, R. Dittmann, L. Ravelli, R. A. Mackie, and W. Egger, Phys. Rev. Lett. 105, 226102 (2010).

[27] S. Wicklein, A. Sambri, S. Amoruso, X. Wang, R. Bruzzese, A. Koehl, and R. Dittmann, Appl. Phys. Lett. 101, 131601 (2012).

[28] R. A. de Souza, F. Gunkel, S. Hoffmann-Eifert, and R. Dittmann, Phys. Rev. B 89, 241401(R) (2014).

[29] A. Koehl, D. Kajewski, J. Kubacki, K. Szot, A. Kuzmin, C. Lenser, P. Meuffels, R. Dittmann, R. Waser, and J. Szade, Phys. Chem. Chem. Phys. 15, 8311 (2013).

[30] C. Lenser, M. Patt, S. Menzel, A. Köhl, C. Wiemann, C. M. Schneider, R. Waser, and R. Dittmann, Adv. Funct. Mater. 24, 4466 (2014).

[31] R. Schafranek, S. Payan, M. Maglione, and A. Klein, Phys. Rev. B 77, 195310 (2008).

[32] T. Fujii, M. Kawasaki, A. Sawa, Y. Kawazoe, H. Akoh, and Y. Tokura, Phys. Rev. B 75, 165101 (2007).

[33] A. A. Sirenko, C. Bernhard, A. Golnik, A. M. Clark, J. Hao, W. Si, and X. X. Xi, Nature (London) 404, 373 (2000).

[34] M. Ahrens, R. Merkle, B. Rahmati, and J. Maier, Physica B (Amsterdam, Neth.) 393, 239 (2007).

[35] J. Sonand S. Stemmer, Phys. Rev. B 80, 035105 (2009).

[36] B. T. Phanand J. Lee, Appl. Phys. Lett. 94, 232102 (2009).

[37] W. Liu, G.-Y. Yang, and C. A. Randall, Jpn. J. Appl. Phys. 48, 051404 (2009).

[38] P. C. Joshiand S. B. Krupanidhi, J. Appl. Phys. 73, 7627 (1993). 